\title{
On the Possible Variations of the Hubble Constant with Distance
}

\author{
Xiang-Ping $\mathrm{Wu}$ \\ Department of Physics, University of Arizona, Tucson, AZ 85721 and \\ Beijing Astronomical Observatory, Chinese Academy of Sciences, Beijing 100080, China \\ Bo Qin \\ Beijing Astronomical Observatory, Chinese Academy of Sciences, Beijing 100080, China \\ and \\ Li-Zhi Fang \\ Department of Physics, University of Arizona, AZ 85721
}

Received 23 October 1995; $\quad$ accepted 9 April 1996 


\begin{abstract}
Current measurements of the Hubble constant $H_{0}$ on scale less than $\sim 100$ Mpc appear to be controversial, while the observations made at high redshift seem to provide a relatively low value. On the other hand, the Hubble expansion is driven by the matter content of the universe. The dynamical analysis on scale of a few $\sim 10 \mathrm{Mpc}$ indicates that the matter density $\Omega_{0}$ is only $\sim 0.2-0.3$, which is significantly smaller than $\Omega_{0}=1$ predicted in the standard inflation model. This might support the tendency of a decreasing Hubble constant towards distance. In this paper, we discuss the influence of a possible variant Hubble constant on two fundamental relations in astronomy: the magnitude-redshift $(m-z)$ and the number-magnitude relations. Using a distant type Ia supernova at $z=0.458$, we show that the deceleration parameter $q_{0}$ or $\Omega_{0}$ cannot be determined from the $m-z$ relation at moderate/high redshift unless the variation of the Hubble constant is a priori measured. It is further demonstrated that the number density of distant sources would be underestimated when their local calibration is employed, which may partially account for the number excess of the faint blue galaxies observed at moderate/high redshift.
\end{abstract}

Subject headings: cosmology: distance scale - large-scale structure of universe 


\section{Introduction}

The Hubble constant $H_{0}$ is an indicator of the global expansion of the universe. Theoretically, this parameter is defined in terms of the Cosmological Principle, i.e., the overall matter distribution of the universe is assumed to be isotropic and homogeneous. The isotropy of the universe has been remarkably demonstrated by the measurement of the $3^{\circ} \mathrm{K}$ microwave background radiation (e.g. Bennett et al. 1996). However, observations have indicated that there exists an inhomogeneous matter distribution in the local universe on scale up to $\sim 100 \mathrm{Mpc}$, such as matter clumps, voids, filaments, etc., which may cause a deviation of the local Hubble expansion from the global one. The pioneering work to directly measure the variation of the Hubble constant with distance was carried out by Sandage, Tammann and Hardy in 1972, using the first ranked E galaxies. Two decades later, Lauer \& Postman (1992) conducted another direct observation from the brightest cluster galaxies to $z=0.05$. These authors have essentially reached a similar conclusion that the ratio of the local Hubble constant $H_{L}$ to the global one $H_{G}$ is consistent with unity, indicative of a very minor effect of local matter clumps and voids on the expansion of the universe. Other evidences from the study of the Hubble diagram of various objects (e.g. Sandage \& Hardy 1973; Tammann \& Sandage 1995) supported that the Hubble constant is roughly invariant.

However, this situation has been recently challenged by several determinations of the Hubble constant utilizing Cepheid variables and the globular cluster luminosity function in some nearby galaxies, which yield a large value of $H_{0}$ ranging from 69 to $87 \mathrm{~km} \mathrm{~s}^{-1} \mathrm{Mpc}$ (Pierce et al. 1994; Freedman et al. 1994; Tanvir et al. 1995; Whitmore et al. 1995). These correspond to an age of the universe of 8-13 Gyr in the frame of the standard cosmological model, while the oldest known stars in globular clusters of our Galaxy are estimated to be about $15.8 \pm 2.1$ Gyr (Bolte \& Hogan 1995). If these calibrations are correct rather than 
suffer from the systematic and logical errors as were argued by Sandage and collaborators (Sandage 1996; Sandage \& Tammann 1996; reference therein), there are two possibilities to remove the apparent conflict over the age of the universe: (1) a nonzero cosmological constant $\lambda_{0} \equiv \Lambda /\left(3 H_{0}^{2}\right)$ [see Ostriker \& Steinhardt (1995) for a summary] and (2) a local low-density region embedded in a globally flat universe (Turner, Cen \& Ostriker 1992; Wu et al. 1995). The later requires a considerably variation of the Hubble constant with distance.

The claim for a deviation of the local Hubble flow from the global one is indirectly supported by the following observations: From the time delay of 1.1 years revealed by over 10 years coverage of the monitoring of the gravitationally-doubled images QSO0957+561A,B at redshift of 1.41, one has found $H_{G}=48_{-7}^{+16} \mathrm{~km} \mathrm{~s}^{-1} \mathrm{Mpc}^{-1}$ [see Wu (1996) for a recent review]. This global value is relatively low though large uncertainties may exist in the resulting $H_{G}$ due to the modeling of the deflectors. For instance, a new lensing model of QSO0957+561A,B based on the same data yields $H_{G} \approx 82.5 \mathrm{~km} \mathrm{~s}^{-1} \mathrm{Mpc}^{-1}$ (Grogin \& Narayan 1996). Another evidence comes from the measurement of Sunyaev-Zel'dovich (S-Z) effect, which is the spectral distortion of the cosmic background radiation due to the inverse Compton cooling of the hot X-ray gas in clusters of galaxies (Sunyaev \& Zel'dovich 1972). It has been found that a relatively small value of the Hubble constant is derived from distant galaxy cluster, e.g. $H_{0}=65 \pm 25 \mathrm{~km} \mathrm{~s}^{-1} \mathrm{Mpc}^{-1}$ for A2218 ( $\left.z=0.171\right)$ (Jones et al. 1993; Birkinshaw \& Hughes 1994), $H_{0}=47 \pm 17 \mathrm{~km} \mathrm{~s}^{-1} \mathrm{Mpc}^{-1}$ for A665 $(z=0.182)$ and $H_{0}=41_{-12}^{+15} \mathrm{~km} \mathrm{~s}^{-1} \mathrm{Mpc}^{-1}$ for Cl0016+16 $(z=0.545)$ (Yamashita 1994). However, the detection of the S-Z effect for nearby Coma cluster provides $H_{0}=71_{-25}^{+30} \mathrm{~km} \mathrm{~s}^{-1} \mathrm{Mpc}^{-1}$ (Herbig et al. 1995). It seems likely that the Hubble constant decreases with redshift. Yet, the errors in these measurements are still large and uncertainties from modeling of the hot X-ray gas in clusters of galaxies need to be further studied (Inagaki, Suginohara \& Suto 1995; Rephaeli 1995). 
On the other hand, it is crucial to realize that the Hubble constant depends on the matter distribution since the expansion is uniquely governed by the gravitational matter of the universe. There have been increasing observational evidences that the local matter density $\Omega_{L}$ within a few $10 \mathrm{Mpc}$ is only $\Omega_{L} \approx 0.2-0.3$ (Bahcall, Lubin \& Dorman 1995; references therein). However, the standard inflation cosmological model predicts an overall matter density of $\Omega_{G} \approx 1$. This matter discrepancy implies a gradient of the Hubble constant along distance.

The present measurement of the Hubble constant as well as the density parameter is still a controversial issue. It turns out to be unclear whether or not the Hubble constant alters with distance. In this paper, we address the following question: If the Hubble constant is variant, what effect will it have on the fundamental relations in astronomy ? In particular we will demonstrate this effect on the magnitude-redshift relation using the distant type Ia supernova (section 2) and on the source number counts using the faint galaxy population (section 3). In section 4 we will construct a simple analytic model for a locally low and globally high density universe instead of the usual simulation method (e.g. Turner et al. 1992; Suto et al. 1995; Nakamura \& Suto 1995; Shi et al. 1995). A brief discussion and our conclusions will be given in section 5 .

\section{Comparison of nearby and distant supernovae}

Study of the magnitude-redshift relation $(m-z)$, i.e. the Hubble diagram, at moderate and high redshift has been thought to be able to provide information on the deceleration parameter $q_{0}$ or $\Omega_{0}$. However, to do this one actually needs to presume that $H_{0}$ is invariant with redshift. In the past decades many attempts have been made to derive $\Omega_{0}$ from the $m-z$ relation for various objects. We will illustrate this procedure and discuss the influence of the variation of $\Omega_{0}$ or $H_{0}$ on $m-z$ relation using the most distant type Ia supernova (SN 
Ia) discovered recently at $z=0.458$ (Perlmutter et al. 1995).

Because of the narrow distribution of their absolute magnitudes at maximum light, SN Ia's are often considered to be "standard candles" and therefore, used as distance indicators (Branch \& Tammann 1992; Branch, Nugent \& Fisher 1996 and references therein). The detection of $\sim 20$ distant SN Ia's at redshift $z \approx 0.35-0.5$ provides an unprecedented method of measuring the geometry of distant universe (Perlmutter et al. 1995; Goobar \& Perlmutter 1995; Branch et al. 1996). Neglecting the $K$ correction and the extinction of our Galaxy for the moment, the $m-z$ relation of a SN Ia with absolute magnitude $M$ is

$$
m=M+5 \log \left(D_{L} / 10 \mathrm{pc}\right)
$$

where $D_{L}$ is the luminosity distance:

$$
D_{L}=\left(\frac{2 c}{H_{0}}\right) \frac{z \Omega_{0}+\left(\Omega_{0}-2\right)\left(-1+\sqrt{\Omega_{0} z+1}\right)}{\Omega_{0}^{2}} .
$$

If the absolute magnitude $M_{\max }$ at maximum light is calibrated using the nearby SN Ia's, e.g. for $B$ band (Perlmutter et al. 1995),

$$
M_{\max }-5 \log \left(H_{0} / 75\right)=-18.86 \pm 0.06
$$

then the monitoring of the apparent magnitude variation of distant SN Ia's can give rise to the mean mass density of the universe, $\Omega_{0}$. Calibrating the $B$ light curve of a distant SN Ia at $z=0.458$ by eq.(1) and the template standard model for nearby SN Ia's, Perlmutter et al. (1995) reached a result of $\Omega_{0} \approx 0.2$. At the time of the refereeing process, another 20 distant SN Ia's have been found and a similar inverstigation has been made by the LBL group (Perlmutter et al. 1996; Kim et al. 1996), which leads to a new determination of $\Omega_{0} \approx 1$. Note that their analysis was based on the hypothesis that the local expansion rate $H_{L}$ remains as the same as the global Hubble flow $H_{G}$ so that the Hubble constant does not appear in their final result. 
What would happen if $H_{L} \neq H_{G}$ ? In a locally low but globally high density universe $\left(H_{L}>H_{G}\right)$, the observed apparent magnitude of a distant SN Ia at maximum light should be generally written as

$$
m_{\max }=\left(M_{\max }-5 \log \frac{H_{L}}{75}\right)-5 \log \frac{H_{G}}{H_{L}}+5 \log \frac{\Omega_{0} z+\left(\Omega_{0}-2\right)\left(\sqrt{1+\Omega_{0} z}-1\right)}{\Omega_{0}^{2}}+5 \log \frac{2 c}{75},
$$

where the first term on the right hand side is the local calibration at maximum light and the second one denotes the deviation of the local expansion from the global Hubble flow. Compared with the conventional Mattig's relation, eq.(4) contains two free parameters: $\Omega_{0}$ and $H_{L} / H_{G}$. Taking the observational data from the first discovered distant SN Ia, $m_{R, \max }=22.2, A_{R}=0.006$ ( $R$ extinction) and $\triangle m_{R B}=-0.7$ (difference of $R$ and $B$ magnitudes) (Perlmutter et al. 1995), we have plotted the variation of $H_{L} / H_{G}$ versus $\Omega_{0}$ in Fig.1, together with the total errors introduced by the intrinsic dispersion of $M$ for the SN Ia $( \pm 0.25)$ and other errors $( \pm 0.16)$ from the photo noise, calibration, etc. The major error stems from the intrinsic dispersion, which can be greatly reduced with the employment of more distant SN Ia's. Based on one distant SN Ia at $z=0.458$, limit on the variation of the Hubble constant is $H_{L} / H_{G}=1.02_{-0.17}^{+0.22}$ and $1.12_{-0.19}^{+0.23}$ for a global density of $\Omega_{G}=0.2$ and $\Omega_{G}=1$, respectively. Because of the large errorbars, the variation of $H_{L} / H_{G}$ has not been well constrained. However, this example shows that the $m-z$ relation cannot be used for the determination of the global $\Omega_{G}$ unless the variation of the Hubble constant is a priori given.

EDITOR: PLACE FIGURE 1 HERE.

\section{Comparison of nearby and distant galaxies}

The deviation of $H_{L}$ from $H_{G}$ also affects the calibration of the number-magnitude relation. Compared with the local sources (e.g. galaxies), the luminosity distance $D_{L}$ and 
the volume element in the distant universe change by a factor of $H_{L} / H_{G}$ and $\sim\left(H_{L} / H_{G}\right)^{3}$, respectively. As a result, the number density of the sources at high redshift would appear to be different from the local one.

We now show this effect by considering the galaxy number counts. We employ the Schechter luminosity function for the galaxy luminosity distribution which has been calibrated locally (Efstathiou, Ellis \& Peterson 1988): $\phi^{*}=1.56 \times 10^{-2} h_{L}^{3} \mathrm{Mpc}^{-3}$, $\alpha=-1.11$ and $M^{*}=-19.6+5 \log h_{L}$ where $h_{L}=H_{L} / 100 \mathrm{~km} \mathrm{~s}^{-1} \mathrm{Mpc}^{-1}$. We keep the same galaxy morphological composition as those used by Yoshii \& Sato (1992): $(\mathrm{E} / \mathrm{S} 0, \mathrm{Sab}, \mathrm{Sbc}, \mathrm{Scd}, \mathrm{Sdm})=(0.215,0.185,0.160,0.275,0.165)$ and take the $K$ correction from King \& Ellis (1985). Because our goal is to demonstrate the influence of $H_{L} / H_{G}$, we will not include the evolution correction which is dependent of the cosmological models $\left(H_{0}\right.$ and $\left.q_{0}\right)$. Furthermore, we assume a constant comoving number density of galaxies and extrapolate the above conditions up to $z=3$. Fig.2 shows the differential number counts of galaxies per square degree in $B$ band for $H_{L}=H_{G}$ and $H_{L}=1.3 H_{G}$, respectively, where the global matter density is chosen to be unity. It turns out that there are no apparent differences in the curves at the bright limiting magnitude. However, a significant excess of faint population is predicted for $H_{L}>H_{G}$. Note that the scale in the $m$-axis should not be regarded as an accurate indicator of the magnitude due to the ignorance of the color and evolution corrections.

\section{EDITOR: PLACE FIGURE 2 HERE.}

This number disprepancy of faint galaxies arising from the local calibration of the Hubble constant may partially explain the excess of number counts of faint blue galaxies in observations (Broadhurst, Ellis \& Glazebrook 1992; references therein). Meanwhile, this might resolve the too few giant luminous arcs predicted from the statistical lensing 
by clusters of galaxies, in which background galaxies were assumed to follow their local properties (Wu \& Hammer 1993; Wu \& Mao 1996). Arcs/arclets have been shown to be a very efficient tool for probe of the distant galaxies [see Fort \& Mellier (1994) for a recent review]. Alternatively, the magnification probability of distant quasars by foreground galaxies (Turner, Ostriker \& Gott 1984) is increased by a factor of roughly $\left(H_{L} / H_{G}\right)^{3}$ while the expected separation of images remains unchanged.

\section{Modeling of the local universe}

While the dynamical measurement shows $\Omega_{L} \approx 0.2-0.3$ (Bahcall et al. 1995), we are situated in a low-density region if $\Omega_{G}$ is close to unity as predicted by the standard inflation model. We now construct a simple model for the local universe. The Tolman-Bondi metric is suitable for the description of the space-time of a spherical perturbation in an expanding universe. For an initial underdense perturbation $\delta_{i}$ at the cosmic epoch $t=t_{i}$ or at redshift $z_{i}$, its evolution follows

$$
\begin{gathered}
\frac{r}{r_{i}}=\frac{\Omega_{i}\left(1-\delta_{i}\right)}{1-\Omega_{i}\left(1-\delta_{i}\right)} \frac{\cosh \eta-1}{2} \\
\sinh \eta-\eta=\frac{2\left[1-\Omega_{i}\left(1-\delta_{i}\right)\right]^{3 / 2}}{\Omega_{i}\left(1-\delta_{i}\right)}\left(H_{i} t_{i}\right)\left(\frac{t}{t_{i}}-1\right)+\frac{2 \sqrt{1-\Omega_{i}\left(1-\delta_{i}\right)}}{\Omega_{i}\left(1-\delta_{i}\right)}-\cosh ^{-1} \frac{2-\Omega_{i}\left(1-\delta_{i}\right)}{\Omega_{i}\left(1-\delta_{i}\right)}
\end{gathered}
$$

where $\Omega_{i}$ and $H_{i}$ are the mean mass density and the Hubble constant at $t=t_{i}$ for the background universe, respectively, which are related to their present values through

$$
\begin{gathered}
\Omega_{i}=\frac{\Omega_{0}\left(1+z_{i}\right)}{1+\Omega_{0} z_{i}} \\
H_{i}=H_{0}\left(1+z_{i}\right) \sqrt{1+\Omega_{0} z_{i}} .
\end{gathered}
$$

For the sake of simplicity as well as the desire of the inflation model, the background universe is often taken to be flat in the previous similar studies (e.g. Nakamura \& Suto 
1995; Wu et al. 1995). With the choice of $\Omega_{0}=1$ eqs.(5) and (6) reduce to the results of

Wu et al. (1995). We are now dealing with a global open/flat universe. The ages of an open universe at $t=t_{i}$ and $t=t_{0}$ (present) are

$$
t_{i}=\frac{1}{H_{0}}\left(\frac{\sqrt{1+\Omega_{0} z_{i}}}{\left(1-\Omega_{0}\right)\left(1+z_{i}\right)}-\frac{\Omega_{0}}{2\left(1-\Omega_{0}\right)^{3 / 2}} \cosh ^{-1} \frac{2-\Omega_{0}+\Omega_{0} z_{i}}{\Omega_{0}\left(1+z_{i}\right)}\right)
$$

and

$$
t_{0}=\frac{1}{H_{0}}\left(\frac{1}{1-\Omega_{0}}-\frac{\Omega_{0}}{2\left(1-\Omega_{0}\right)^{3 / 2}} \cosh ^{-1} \frac{2-\Omega_{0}}{\Omega_{0}}\right),
$$

respectively. Defining the present Hubble constant as $H_{0} \equiv \dot{r_{0}} / r_{0}$, we can find the relationship between the local Hubble constant $H_{L}$ and the background one $H_{G}$ to be

$$
\frac{H_{L}}{H_{G}}=\frac{\left(1+z_{i}\right) \sqrt{1+\Omega_{i} z_{i}}}{\left(r_{0} / r_{i}\right)} \sqrt{1-\Omega_{i}\left(1-\delta_{i}\right)+\frac{\Omega_{i}\left(1-\delta_{i}\right)}{\left(r_{0} / r_{i}\right)}} .
$$

Finally, the energy conservation gives rise to the present mass density of the local universe $\Omega_{L}$ developed from the initial fluctuation $\delta_{i}$ :

$$
\Omega_{L}=\Omega_{0}\left(1-\delta_{i}\right)\left[\frac{1+z_{i}}{\left(r_{0} / r_{i}\right)}\right]^{3}
$$

Setting the epoch of the initial perturbation of our local low-density region to be the decoupling of $z_{i} \approx 1000$, we have performed the numerical computations of $H_{L} / H_{G}$ versus $\Omega_{0}$ for the local mass density of $\Omega_{L}=0.1,0.2$ and 0.3 and illustrated the results in Fig.3. As is expected, the deviation of the local expansion rate from the global Hubble flow depends critically on the mass density difference between the local and background universe. Using the local dynamical measurement of $\Omega_{L} \approx 0.2-0.3$ (Bahcall et al. 1995), we can set the variation of the Hubble constant in a global flat universe to be $H_{L} / H_{G} \approx 1.3$. Recall that $H_{L} / H_{G}=1.5$ in the extreme case of where $\Omega_{L}=0$ and $\Omega_{G}=1$. 


\section{Discussion and conclusions}

We have shown that if the matter density of our local universe within $\sim 100 \mathrm{Mpc}$ is $\Omega_{L} \approx 0.2-0.3$, as indicated by the dynamical observations (Bahcall et al. 1995), and the global value is close to unity, as predicted by the standard inflation model, then the local Hubble expansion rate would be $\sim 1.3$ times larger than the global one. The current measurements of the Hubble constant do not exclude this variation with distance. As a consequence, we need to be cautious of using some fundamental relations.

Utilizinging a distant SN Ia at $z=0.458$ discovered recently (Perlmutter et al. 1995), we have demonstrated how the variation of $H_{L} / H_{G}$ affects the magnitude-redshift relation. Given the global matter density $\Omega_{G}$, we have placed the preliminary limit on $H_{L} / H_{G}$. It turns out that due to the large intrinsic dispersion for the SN Ia, the variation of $H_{L} / H_{G}$ has not been well constrained. The current limit is consistent with either a uniform expansion or a variation of $H_{L} / H_{G}$. Nevertheless, we have pointed out that there are two free parameters, $\Omega_{G}$ and $H_{L} / H_{G}$, appearing in the $m-z$ relation at moderate/high redshift if the local calibration is used, which would invalidate the conventional method of determining $\Omega_{0}$ from the difference in the Mattig relation unless observations are able to provide the relationship between $H_{L}$ and $H_{G}$. This is consistent with the recent work by Kim et al. (1996).

The deviation of the local Hubble expansion from the global one would have influence on the calibration of the number counts of distant sources. We have found that the number density of distant faint galaxies would be underestimated if $H_{L}>H_{G}$. This may partially remove the demand for a strong evolution scenario of galaxies population in order to account for the number excess of faint (blue) galaxies. As a result, the theoretically predicted number of giant arcs, which are the distorted images of distant galaxies by the gravitational potentials of foreground clusters of galaxies, would increases. This probably 
provides another explanation for the deficit of giant arcs in statistical lensing (Wu \& Mao 1996).

We have only discussed the effect of $H_{L} / H_{G}$ on the magnitude-redshift relation and the number counts. Indeed, if the local expansion is larger than the global one, many aspects of astrophysics might need to be modified. The crucial point depends on the measurements of both the Hubble constant and the matter density at different redshift. The current status on the determinations of $H_{0}$ and $\Omega_{0}$ appears to be unfortunate: The observations using the same method can result in very different results. Here we do not intend to be involved in the disputes. Instead, we point out that it would be of great significance if one would explore other possibilities which can be used to probe or constrain the variations of the Hubble constant and the density parameter.

We are grateful to the referees, Allan Sandage and David Branch, for their constructive comments and suggestions. WXP was supported by the National Science Foundation of China and a World-Laboratory fellowship. 


\section{REFERENCES}

Bahcall, N. A., Lubin, L. M., \& Dorman, V. 1995, ApJ, 447, L81

Bennett, C. L., et al. 1996, ApJ, submitted

Birkinshaw, M., \& Hughes, J. P. 1994, ApJ, 420, 33

Bolte, M., \& Hogan, C. J. 1995, Nature, 376, 399

Branch, D., Nugent, P., \& Fisher, A. 1996, Proc. of NATO ASI on Thermonuclear Supernovae, eds. R. Canal, P. Ruiz-Lapuente \& J. Isern (Dordrecht: Kluwer), in press

Branch, D., \& Tammann, G. A. 1992, ARA\&A, 30, 359

Broadhurst, T. J., Ellis, R. S., \& Glazebrook, K. 1992, Nature, 355, 55

Efstathiou, G., Ellis, R. S., \& Peterson, B. A. 1988, MNRAS, 232, 431

Fort, B., \& Mellier, Y. 1994, A\&A Rev., 5, 239

Freedman, W. L., et al. 1994, Nature, 371, 757

Goobar, A., \& Perlmutter, S. 1995, ApJ, 450, 14

Grogin, N. A., \& Narayan, R. 1996, ApJ, submitted

Herbig, T., Lawrence, C. R., \& Readhead, A. C. S. 1995, ApJ, 449, L5

Inagaki, Y., Suginohara, T., \& Suto, Y. 1995, PASJ, 47, 411

Jones, M., et al. 1993, Nature, 365, 320

Kim, A. et al. 1996, Proc. of NATO ASI on Thermonuclear Supernovae, eds. R. Canal, P. Ruiz-Lapuente \& J. Isern (Dordrecht: Kluwer), in press

King, C. R., \& Ellis, R. S. 1985, ApJ, 288, 456

Lauer, T. R., \& Postman, M. 1992, ApJ, 400, L47 
Nakamura, T. T., \& Suto, Y. 1995, ApJ, 447, L65

Ostriker, J. P., \& Steinhardt, P. J. 1995, Nature, 377, 600

Perlmutter, S., et al. 1995, ApJ, 440, L41

Perlmutter, S., et al. 1996, Proc. of NATO ASI on Thermonuclear Supernovae, eds. R. Canal, P. Ruiz-Lapuente \& J. Isern (Dordrecht: Kluwer), in press

Pierce, M. J., et al. 1994, Nature, 371, 385

Rephaeli, Y. 1995, ARA\&A, 33, 541

Sandage, A. 1996, private communication

Sandage, A., \& Hardy, E. 1973, 183, 743

Sandage, A., \& Tammann, G. A. 1996, ApJ, submitted

Sandage, A., Tammann, G. A., \& Hardy, E. 1972, ApJ, 172, 253

Shi, X., Widrow, L. M., \& Dursi, L. J. 1995, preprint

Sunyaev, R. A., \& Zel'dovich, Y. B. 1972, Comm. Astrophys. Space Sci., 4, 173

Suto, Y., Suginohara, T., \& Inagaki, Y. 1995, Prog. Theor. Phys. Phys., 93, 839

Tammann, G. A., \& Sandage, A. 1995, ApJ, 452, 16

Tanvir, N. R., Shanks, T., Ferguson, H. C., \& Robinson, D. R. T. 1995, Nature, 377, 27

Turner, E. L., Cen, R., \& Ostriker, J. P. 1992, AJ, 103, 1427

Turner, E. L., Ostriker, J. P., \& Gott III, H. R. 1984, ApJ, 284, 1

Whitmore, B. C., Sparks, W. B., Lucas, R. A., Macchetto, F. D., \& Biretta, J. A. 1995, ApJ, 454, L73

Wu, X. P. 1996, Fund. of Cos. Phys., in press

Wu, X. P., Deng, Z., Zou, Z., Fang, L. Z., \& Qin, B. 1995, ApJ, 448, L65 
Wu, X. P., \& Hammer, F. 1993, MNRAS, 262, 187

Wu, X. P., \& Mao, S. 1996, ApJ, in press

Yamashita, K. 1994, New Horizon of X-Ray Astronomy: First Results from ASCA, eds. F. Makino \& T. Ohashi (Tokyo: Universal Academy Press), 279

Yoshii, Y., \& Sato, K. 1992, ApJ, 387, L7 
Fig. 1.- Limit on the variation of the Hubble constant $H_{L} / H_{G}$ against the global mass density $\Omega_{0}$ using the distant type Ia supernova at $z=0.458$. The solid line corresponds to the peak magnitude $m_{R}=22.2$ and the dashed lines are the error bars arising from the intrinsic dispersion $( \pm 0.25)$ of the local type Ia supernovae and the measurement uncertainties at maximum light $( \pm 0.16)$ (The supernova data are taken from Perlmutter et al. 1995).

Fig. 2.- Differential number counts of galaxies in $B$ band per square degree for an uniform Hubble flow $H_{L}=H_{G}$ and a variable Hubble constant $H_{L}=1.3 H_{G}$, respectively. Evolution effect has not been included.

Fig. 3.- Variations of the Hubble expansion rate $\left(H_{L} / H_{G}\right)$ against the global mean mass density of the universe $\Omega_{0}$ for a given local mass density $\Omega_{L}$. 


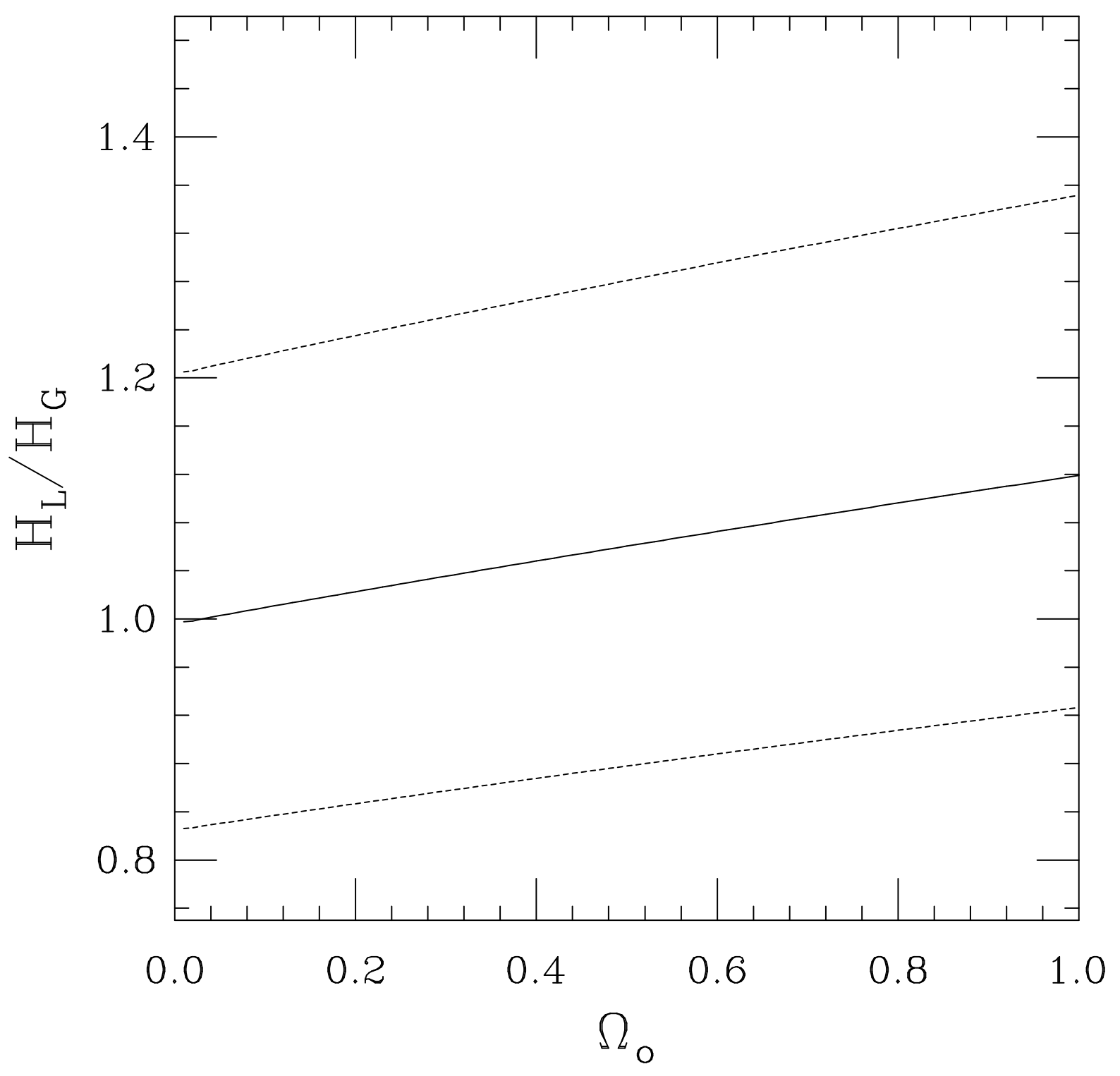




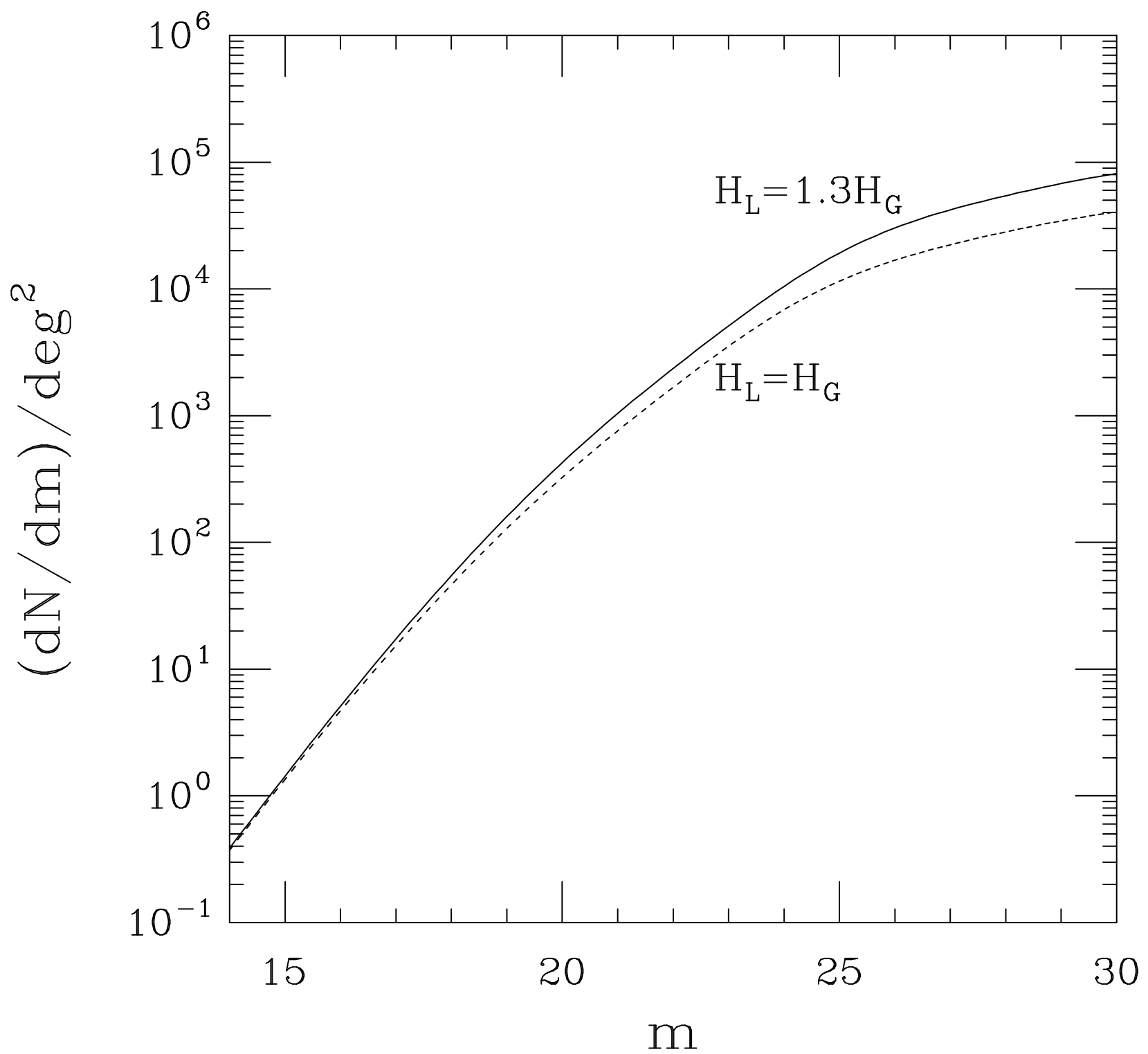




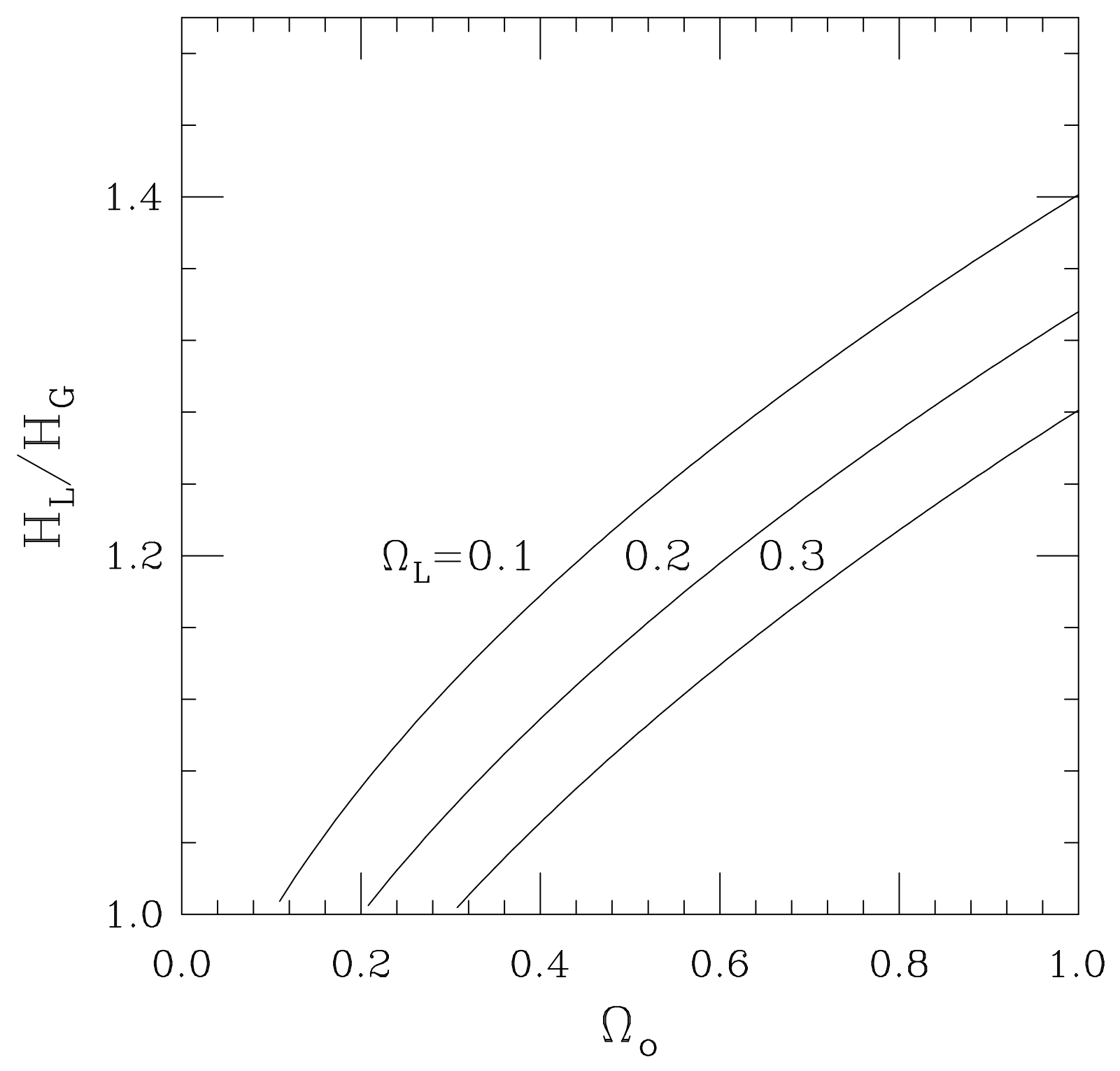

